\title{
Prognostic significance of preoperative serum CEA in primary mucinous ovarian carcinoma: a retrospective cohort study
}

This article was published in the following Dove Press journal:

Cancer Management and Research

\author{
Wei Lin \\ Dongyan Cao \\ Keng Shen \\ Department of Obstetrics and \\ Gynecology, Peking Union Medical \\ College Hospital, Chinese Academy \\ of Medical Sciences and Peking Union \\ Medical College, Beijing, People's \\ Republic of China
}

\begin{abstract}
Purpose: The purpose of this study was to evaluate the prognostic significance of preoperative serum carcinoembryonic antigen (CEA) in primary mucinous ovarian carcinoma (MOC). Patients and methods: We reviewed 57 patients with primary MOC, in whom preoperative serum CEA had been measured. All patients were treated at Peking Union Medical College Hospital between June 1996 and September 2016.

Results: Preoperative serum CEA was elevated $(>5.0 \mathrm{ng} / \mathrm{mL})$ in 10 patients $(17.5 \%)$, with a median serum CEA of $9.6 \mathrm{ng} / \mathrm{mL}(5.4-111.7 \mathrm{ng} / \mathrm{mL})$. CEA was significantly associated with preoperative serum cancer antigen $125(P=0.002)$, surgical debulking status $(P=0.015)$, and tumor stage $(P=0.001)$. Univariate analysis showed that patients with elevated CEA had significantly worse overall survival (OS) than patients with normal CEA (5-year OS: 50.8\% vs $91.9 \%$, respectively; $P=0.013$ ), but there was no significant difference in progression-free survival between the two groups $(P=0.307)$. Multivariate analysis further demonstrated that advanced stage was an independent prognostic predictor for OS ( $P=0.002$, HR: $15.925,95 \%$ CI: 2.745-92.404).
\end{abstract}

Conclusion: Elevated preoperative serum CEA was strongly correlated with advanced stage in primary $\mathrm{MOC}$, which may indicate a poorer prognosis. Further investigation of the intrinsic relationship between CEA and primary MOC is now required.

Keywords: epithelial ovarian cancer, mucinous carcinoma, tumor marker, carcinoembryonic antigen, prognosis

\section{Introduction}

Mucinous ovarian carcinoma (MOC) is a relatively rare entity among the different histological subtypes of epithelial ovarian cancer (EOC). Primary MOC accounts for approximately $2 \%-3 \%$ of EOC cases. ${ }^{1,2}$ Although early-stage MOC presents with a good prognosis, the survival of patients with advanced-stage MOC is worse than that of other EOC subtypes, especially those afflicted by the serous subtype (5-year survival: $10.2 \%-25.7 \%$ vs $20.3 \%-33.6 \%$, respectively). ${ }^{3-5}$ Therefore, the identification of prognostic factors may facilitate the decision-making process for MOC patients and thus improve their clinical outcomes.

Serum cancer antigen 125 (CA125) has been routinely used to monitor the response of patients with EOC to chemotherapy, disease progression, and recurrence. ${ }^{6,7}$ In MOC, however, the association between preoperative serum CA125 and survival is weak. ${ }^{8}$ In fact, carcinoembryonic antigen (CEA) level is elevated more frequently than CA125 in patients with $\mathrm{MOC},{ }^{9,10}$ rendering CEA a particularly promising candidate for prognostic prediction. An increasing number of studies have suggested that pretreatment

\footnotetext{
Correspondence: Dongyan Cao

Department of Obstetrics and Gynecology, Peking Union Medical College Hospital, Chinese Academy of Medical Sciences and Peking Union Medical College, No I Shuaifuyuan, Wangfujing, Dongcheng District, Beijing 100730, People's Republic of China

Tel +86 1069156114

Fax +861068419898

Email caodongyan@pumch.cn
} 
serum CEA level is an independent prognostic factor for colorectal cancer, breast cancer, non-small cell lung cancer, and gastric cancer. ${ }^{11-14}$ However, due to the rarity of this disease, the prognostic significance of preoperative CEA level in patients with MOC remains unknown. In this study, we aimed to evaluate the prognostic value of preoperative CEA elevation in patients with primary MOC.

\section{Patients and methods Study population}

We reviewed data of all MOC patients treated at Peking Union Medical College Hospital (PUMCH) between 1996 and 2016. In our institute, review of digestive symptoms and fecal occult blood tests were performed routinely in patients with pelvic mass, to exclude the possibility of gastrointestinal tract origin. Gastrointestinal endoscopy was performed in suspected cases. Postoperatively, immunohistochemistry staining tests were further performed to distinguish cases of primary MOC from metastatic MOC. According to the 2014 WHO Classification of Tumors of Female Reproductive Organs, ${ }^{15}$ primary MOC cases are often positive for cytokeratin 7 (CK7) and cytokeratin 20 (CK20; 75\% of cases), while colorectal and appendiceal tumors are usually negative for CK7 and positive for CK20. CK7 positivity and CK20 negativity favor the diagnosis of primary MOC ( $25 \%$ of cases), pancreatobiliary cancer, and gastric cancer. ${ }^{16}$ In addition, the immunostaining of paired box gene 8 (PAX8) and caudal-related homeobox transcription factor 2 (CDX2) can sometimes be useful. PAX8 is positive in $\sim 50 \%$ of primary MOC cases but negative in patients with colorectal, appendiceal, pancreatobiliary, and gastric cancers. ${ }^{15} \mathrm{CDX} 2$ expression in primary ovarian mucinous tumors $(40 \%)$ is lower than that in metastatic carcinomas of gastrointestinal tract origin $(90 \%) .{ }^{17}$ In the present study, we focused our attention on patients with primary MOC who had preoperative serum CEA values recorded in their medical records. Patients were excluded if they had metastatic MOC or mixed EOC subtypes.

A range of data were acquired from medical records, including age, preoperative serum CEA and CA125 values, types of surgery, surgical debulking status, laterality of disease, tumor size, stage, lymph node metastasis status, and adjuvant chemotherapy. All identifying patient data were anonymized. Optimal surgical debulking was defined as when the residual disease was $\leq 1 \mathrm{~cm}$ in size. Tumor size of ovarian or fallopian tube lesions was measured intraoperatively by gynecologists and recorded in the surgical note. Disease stage was reassessed according to the 2014 International Federation of Gynecology and Obstetrics (FIGO) staging system. ${ }^{18}$
Following initial surgery, platinum-based chemotherapy was administered to patients in accordance with National Comprehensive Cancer Network guidelines. ${ }^{19-22}$

All patients were followed-up every 3 months for the first year after the completion of primary therapy, then every 6 months during the period between the second to the fifth year, and annually thereafter. At each follow-up visit, we reviewed symptoms, carried out a pelvic examination, measured serum tumor markers, and performed an ultrasound/computed tomography scan (if indicated). The last patient follow-up appointment was in October 2018.

\section{Serum CEA analysis}

Venous blood samples ( $3 \mathrm{~mL}$ per patient) were obtained within 2 weeks before the primary surgery for preoperative CEA measurement. Serum CEA levels were evaluated using an automatic electrochemistry luminescence immunoassay system (Cobas E601; Hoffman-La Roche Ltd., Basel, Switzerland). Normal CEA values should be $\leq 5.0 \mathrm{ng} / \mathrm{mL}$.

\section{Statistical analyses}

All statistical analyses were performed using the IBM SPSS Statistics for Mac OS Package, Version 24.0 (IBM Corporation, Armonk, NY, USA). Categorical variables were compared by the Pearson chi-squared test or Fisher's exact test. The Kaplan-Meier method was used to generate survival curves, and the log-rank test was used for univariate survival analysis. In order to identify independent prognostic factors, we performed multivariate analysis using the Cox proportional hazards model. Variables that were statistically significant in the univariate analysis were then included in the multivariate analysis. Progression-free survival (PFS) was calculated from the date of diagnosis to the date of tumor recurrence, progression, or death. Overall survival (OS) was calculated from the date of diagnosis to the date of the last follow-up or death. A $P$-value $<0.05$ was considered to be statistically significant.

\section{Ethics approval}

The study was approved by the Ethics Committee of PUMCH, and written informed consent for this retrospective study was formally waived by the Ethics Committee. All procedures performed in this study were in accordance with the Declaration of Helsinki.

\section{Results}

\section{Patient characteristics}

A total of 57 consecutive primary MOC patients with available preoperative serum CEA values were included in our 
Table I Clinicopathological characteristics of 57 patients with primary MOC

\begin{tabular}{|c|c|c|}
\hline Characteristics & Patients, $\mathbf{n}^{\mathbf{a}}$ & $\%$ \\
\hline Median age (range), years & $30(18-74)$ & \\
\hline \multicolumn{3}{|l|}{ Preoperative serum CEA (ng/mL) } \\
\hline$\leq 5.0$ & 47 & 82.5 \\
\hline$>5.0$ & 10 & 17.5 \\
\hline \multicolumn{3}{|l|}{ Preoperative serum CA I 25 (U/mL) } \\
\hline$\leq 35$ & 30 & 53.6 \\
\hline$>35$ & 26 & 46.4 \\
\hline \multicolumn{3}{|l|}{ CSS } \\
\hline TH+BSO+omentectomy+appendectomy & 35 & $6 \mathrm{I} .4$ \\
\hline USO+omentectomy+appendectomy & 22 & 38.6 \\
\hline \multicolumn{3}{|l|}{ Surgical debulking status } \\
\hline Optimal & 53 & 93.0 \\
\hline Suboptimal & 4 & 7.0 \\
\hline \multicolumn{3}{|l|}{ Laterality of disease } \\
\hline Unilateral & 52 & 91.2 \\
\hline Bilateral & 5 & 8.8 \\
\hline Tumor size, mean (range), cm & $15(2-40)$ & \\
\hline \multicolumn{3}{|l|}{ FIGO stage } \\
\hline IA & 17 & 29.8 \\
\hline IC & 25 & 43.9 \\
\hline$\|$ & 3 & 5.2 \\
\hline III & 12 & 21.1 \\
\hline \multicolumn{3}{|l|}{ Lymph node metastasis status } \\
\hline Yes & 2 & 4.2 \\
\hline No & 46 & 95.8 \\
\hline \multicolumn{3}{|l|}{ Adjuvant chemotherapy } \\
\hline Yes & 44 & 77.2 \\
\hline No & 13 & 22.8 \\
\hline
\end{tabular}

Note: ${ }^{\mathrm{a}}$ Data on preoperative serum CAI 25 and lymph node metastasis status were available for 56 and 48 patients, respectively.

Abbreviations: $\mathrm{BSO}$, bilateral salpingo-oophorectomy; CAI25, cancer antigen 125; CEA, carcinoembryonic antigen; CSS, comprehensive staging surgery; FIGO, International Federation of Gynecology and Obstetrics; MOC, mucinous ovarian carcinoma; TH, total hysterectomy; USO, unilateral salpingo-oophorectomy.

study. Patient characteristics are given in Table 1 . The median age at the time of diagnosis was 30 years (range: 18-74 years). Preoperative serum CEA was elevated $(>5.0 \mathrm{ng} / \mathrm{mL})$ in $10(17.5 \%)$ patients. The median preoperative serum CEA level in these patients was $9.6 \mathrm{ng} / \mathrm{mL}$ (range: $5.4-111.7 \mathrm{ng} /$ $\mathrm{mL})$. A total of $26(46.4 \%)$ patients had elevated preoperative levels of serum CA125 (>35 U/mL).

All patients underwent comprehensive staging surgery (CSS). A total of 35 (61.4\%) patients received total hysterectomy, bilateral salpingo-oophorectomy, omentectomy, and appendectomy (including metastasectomy and multiple biopsies as required). In all, 22 (38.6\%) patients underwent fertility-preserving CSS (including unilateral salpingo-oophorectomy, omentectomy, and appendectomy). Lymphadenectomy was performed in all cases, except for nine $(16.1 \%)$ patients with grossly normal lymph nodes. Optimal surgical debulking was achieved in 53 (93.0\%) patients. In all, $52(91.2 \%)$ patients had unilateral disease. Five $(8.8 \%)$ patients had bilateral tumors, raising suspicion for metastatic MOC. The results of gastrointestinal evaluation and immunohistochemical staining tests provided strong evidence that these five patients were all afflicted by primary MOC rather than metastatic MOC (Table 2). The mean tumor size was $15 \pm 8 \mathrm{~cm}$ (range: $2-40 \mathrm{~cm}$ ). Forty-two, three, and 12 patients had stage I, II, and III diseases, respectively. All stage III patients had peritoneal metastasis, including two patients with retroperitoneal lymph node metastasis. All stage IC-III patients, and five stage IA patients, received adjuvant chemotherapy. The mean number of chemotherapy cycles was $4 \pm 2$ cycles.

During a median follow-up duration of 36 months (range: $2-144$ months), seven (12.3\%) patients experienced recurrence with a median PFS of 14 months (range: 5-43 months). Three patients were platinum resistant. The sites of recurrence included the perihepatic area (5/7), contralateral adnexal area (3/7), peritoneum (3/7), sigmoid (1/7), and hepatic flexure of the colon (1/7). Four stage I patients received repeat cytoreductive surgery and second-line platinum-based chemotherapy after recurrence. One of these patients died 3 months after recurrence because of respiratory and circulatory failure, and the remaining three patients were alive with no evidence of recurrence at the last follow-up visit. One patient with stage IIB and another with stage IIIC disease received only second-line chemotherapy after recurrence, and died of MOC 4 and 5 months after recurrence, respectively. One patient with stage IIC disease was lost to follow-up after recurrence. Six (10.5\%) patients died with a median OS of 16 months (range: 3-45 months), including three recurrent patients and three patients with persistent disease. The 5-year PFS and OS rates for the entire cohort were $82.3 \%$ and $85.5 \%$, respectively.

\section{Preoperative serum CEA values and clinicopathological characteristics}

The frequency of preoperative serum CEA elevation was compared in terms of preoperative serum CA125 $(\leq 35$ $\mathrm{U} / \mathrm{mL}$ vs $>35 \mathrm{U} / \mathrm{mL}$ ), surgical debulking status (optimal vs suboptimal), laterality of disease (unilateral vs bilateral), tumor size $(<10 \mathrm{~cm}$ vs $\geq 10 \mathrm{~cm})$, and FIGO stage (early vs advanced). The results are given in Table 3 . In brief, $34.6 \%(9 / 26)$ of patients with elevated levels of preoperative serum CA125 showed elevated CEA levels, as compared with $3.3 \%(1 / 30)$ of patients with normal CA125 levels $(P=0.002)$. In addition, $75.0 \%(3 / 4)$ of patients with suboptimal debulking had elevated CEA 
Table 2 Results of gastrointestinal evaluation and immunohistochemistry tests in five patients with bilateral tumors

\begin{tabular}{|c|c|c|c|c|c|c|c|c|c|}
\hline \multirow[t]{2}{*}{ Patient } & \multirow{2}{*}{$\begin{array}{l}\text { FIGO } \\
\text { stage }\end{array}$} & \multirow{2}{*}{$\begin{array}{l}\text { Tumor } \\
\text { size }(\mathrm{cm})\end{array}$} & \multicolumn{3}{|l|}{ GI evaluation } & \multicolumn{4}{|c|}{ IHC tests } \\
\hline & & & Symptoms & $\begin{array}{l}\text { Fecal OB } \\
\text { tests }\end{array}$ & $\begin{array}{l}\text { GI } \\
\text { endoscopy }\end{array}$ & CK7 & СК20 & PAX8 & CDX2 \\
\hline 1 & IIIC & 4.0 & $\begin{array}{l}\text { Abdominal pain, } \\
\text { distension }\end{array}$ & - & $N$ & + & $+($ focal) & - & + \\
\hline 2 & IIIC & 6.0 & Abdominal distension & - & $\mathrm{N}$ & ++ & - & 1 & l \\
\hline 3 & IIA & 15.0 & Abdominal distension & - & $\mathrm{N}$ & + & - & + (diffuse) & $+($ focal $)$ \\
\hline 4 & IIIC & 20.0 & - & - & 1 & + & - & 1 & 1 \\
\hline 5 & IIIC & 27.2 & - & - & I & + & + & + & I \\
\hline
\end{tabular}

Notes: "+" indicates positive. "++" indicates strong positive. "-" indicates negative. "l” indicates not estimated.

Abbreviations: CDX2, caudal-related homeobox transcription factor 2; CK7, cytokeratin 7; CK20, cytokeratin 20; FIGO, International Federation of Gynecology and Obstetrics; GI, gastrointestinal; IHC, immunohistochemistry; N, no evidence of malignancy; OB, occult blood; PAX8, paired box gene 8.

Table 3 Preoperative serum CEA values and clinicopathological characteristics in primary MOC

\begin{tabular}{|c|c|c|c|}
\hline \multirow[t]{2}{*}{ Characteristics } & \multicolumn{2}{|c|}{ Preoperative serum CEA } & \multirow[t]{2}{*}{$P$-value ${ }^{c}$} \\
\hline & $\leq 5.0 \mathrm{ng} / \mathrm{mL}(\%)$ & $>5.0 \mathrm{ng} / \mathrm{mL}(\%)$ & \\
\hline \multicolumn{4}{|c|}{ Preoperative serum CAI $25^{a}(\mathrm{U} / \mathrm{mL})$} \\
\hline$\leq 35$ & $29(96.7)$ & I (3.3) & 0.002 \\
\hline$>35$ & $17(65.4)$ & $9(34.6)$ & \\
\hline \multicolumn{4}{|c|}{ Surgical debulking status } \\
\hline Optimal & $46(86.8)$ & $7(13.2)$ & 0.015 \\
\hline Suboptimal & I $(25.0)$ & $3(75.0)$ & \\
\hline \multicolumn{4}{|c|}{ Laterality of disease } \\
\hline Unilateral & $44(84.6)$ & $8(15.4)$ & 0.208 \\
\hline Bilateral & $3(60.0)$ & $2(40.0)$ & \\
\hline \multicolumn{4}{|l|}{ Tumor size $^{\mathrm{b}}(\mathrm{cm})$} \\
\hline$<10$ & $5(83.3)$ & I (16.7) & 0.474 \\
\hline$\geq 10$ & $33(91.7)$ & $3(8.3)$ & \\
\hline \multicolumn{4}{|l|}{ FIGO stage } \\
\hline Early (I-II) & $4 I(9 I . I)$ & $4(8.9)$ & 0.001 \\
\hline Advanced (III-IV) & $6(50.0)$ & $6(50.0)$ & \\
\hline
\end{tabular}

Notes: a Data on preoperative serum CAI 25 were available for 56 patients. ${ }^{b}$ Correlation between tumor size and frequency of CEA elevation was analyzed in only stage I patients. 'Calculated by the Pearson chi-squared test or Fisher's exact test. Bold figures indicate statistically significant, $P<0.05$.

Abbreviations: CA125, cancer antigen 125; CEA, carcinoembryonic antigen; FIGO, International Federation of Gynecology and Obstetrics; MOC, mucinous ovarian carcinoma.

levels, as compared with $13.2 \%(7 / 53)$ of patients with optimal debulking $(P=0.015)$. Furthermore, $50.0 \%(6 / 12)$ of patients with advanced-stage disease had elevated CEA levels, as compared with $8.9 \%$ (4/45) of early-stage patients $(P=0.001)$. There was no significant difference in the frequency of CEA elevation when comparing between patients with unilateral disease and those with bilateral disease $(8 / 52$ vs $2 / 5$, respectively; $P=0.208)$. In stage I patients, no significant difference was observed between patients with a tumor size $<10 \mathrm{~cm}$ and those with a tumor size $\geq 10 \mathrm{~cm}$ ( $1 / 6$ vs $3 / 36$, respectively; $P=0.474)$.

\section{Preoperative serum CEA values and survival}

Among the seven patients with recurrence, two patients (stage IC and stage IIIC, respectively) presented with elevated serum
CEA levels both before primary surgery and at the time of recurrence. The remaining five patients (all with early stage disease) showed persistently normal serum CEA levels during the follow-up period.

In univariate analysis, we analyzed preoperative serum CEA, preoperative serum CA125, fertility-preserving CSS, surgical debulking status, laterality of disease, tumor size, FIGO stage, and adjuvant chemotherapy (Table 4). No significant association was observed between any of these parameters and PFS. Elevated preoperative serum CEA $(P=0.013)$, suboptimal debulking $(P<0.001)$, and advanced stage $(P<0.001)$ were significantly associated with a worse OS. The 5-year OS rate for patients with elevated CEA levels was 50.8\%, while that for patients with normal CEA levels was $91.9 \%$ (Figure 1).

In multivariate analysis of OS, a Cox proportional hazards model (Table 5) was created which featured elevated 
Table 4 Univariate survival analysis of prognostic factors related to PFS and OS ${ }^{a}$

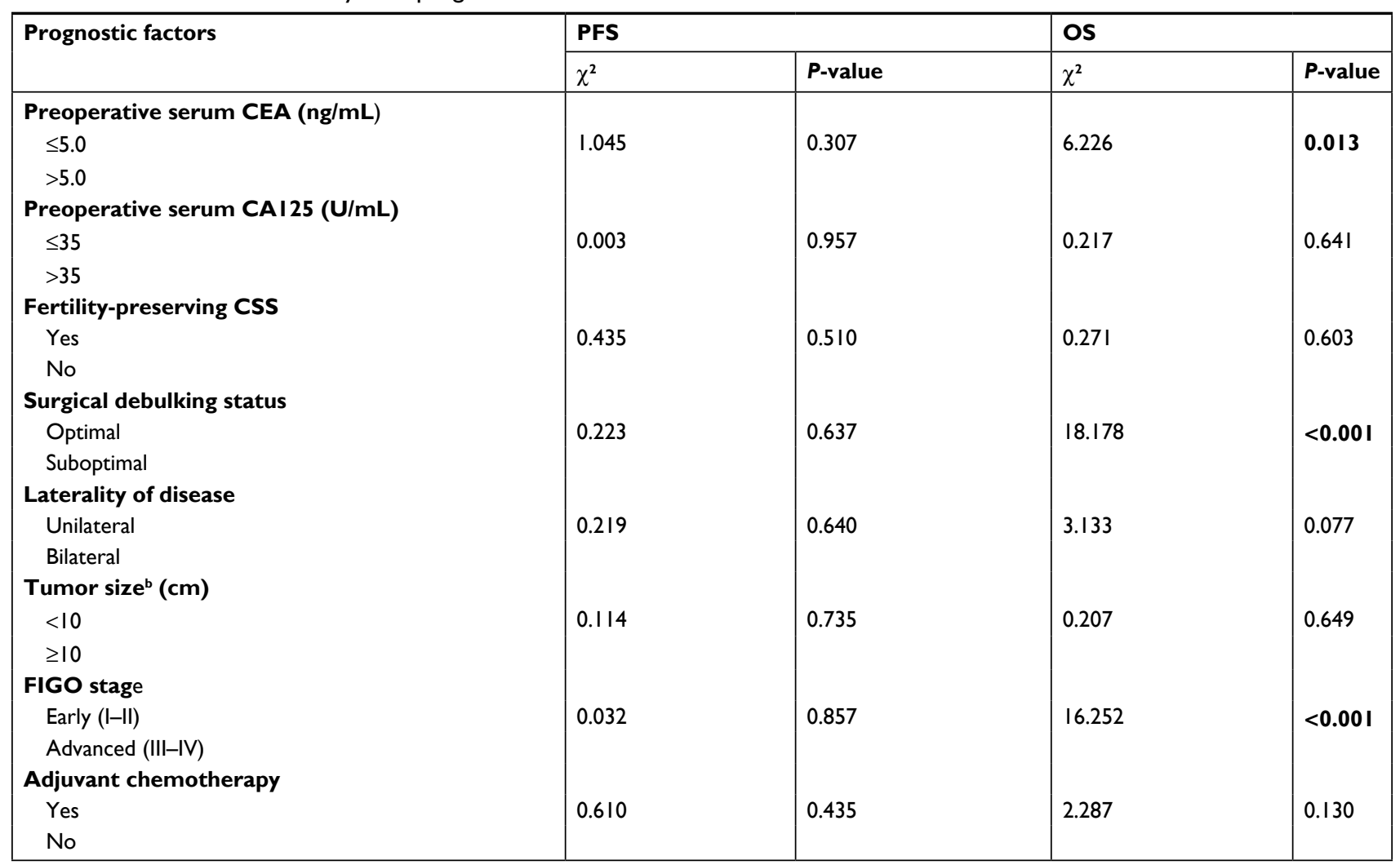

Notes: a Calculated by the log-rank test. bUnivariate survival analysis of tumor size related to PFS and OS was performed in only stage I patients. Bold figures indicate statistically significant, $P<0.05$.

Abbreviations: CAI25, cancer antigen 125; CEA, carcinoembryonic antigen; CSS, comprehensive staging surgery; FIGO, International Federation of Gynecology and Obstetrics; OS, overall survival; PFS, progression-free survival.

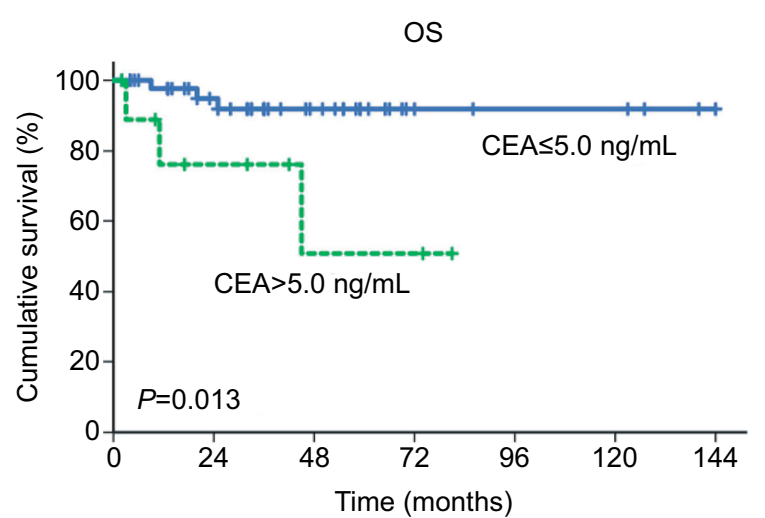

Figure I OS curves of patients with primary ovarian mucinous carcinoma according to preoperative serum CEA values (CEA $\leq 5.0 \mathrm{ng} / \mathrm{mL}$ vs CEA $>5.0 \mathrm{ng} / \mathrm{mL}$ ). Abbreviations: CEA, carcinoembryonic antigen; OS, overall survival.

preoperative serum CEA, suboptimal debulking, and advanced stage. In this model, advanced stage was the only independent prognostic factor for OS (HR: 15.925, 95\% CI: 2.745-92.404, $P=0.002$ ).

\section{Discussion}

Prior to the present study, the prognostic value of preoperative serum CEA in patients with primary MOC remained unclear.
Our analyses showed that patients with elevated CEA levels have significantly lower 5-year OS rates than patients with normal CEA levels (50.8\% vs $91.9 \%$, respectively; $P=0.013$ ), although the multivariate analysis showed that preoperative serum CEA was not an independent prognostic predictor. Instead, advanced stage was identified as an independent predictor of the risk of primary MOC-related death (Figure 2).

Distinguishing between primary MOC and metastatic $\mathrm{MOC}$ is challenging. Metastatic ovarian involvement from other primary sites could be mistakenly reported as primary MOC, especially from colorectal sites. ${ }^{23}$ In a previous study, Seidman et $\mathrm{al}^{24}$ found that in bilateral tumors, $92 \%-95 \%$ of cases are metastatic. This finding has been validated by several other studies. ${ }^{25,26}$ In our present study, five (8.8\%) patients had bilateral tumors, raising suspicion for metastatic MOC. In these patients, gastrointestinal evaluation and immunohistochemistry tests were performed to exclude metastatic MOC (Table 2). It is necessary to carefully differentiate primary MOC from metastatic disease in order to provide optimal treatment for patients.

CEA, first named by Dr Phil Gold and Dr. Samuel Freedman in $1965,{ }^{27}$ has long been recognized as a tumor marker, 
Table 5 Multivariate survival analysis of OS by the Cox proportional hazards model

\begin{tabular}{|l|l|l|l|}
\hline \multirow{2}{*}{ Prognostic factors } & \multicolumn{3}{l|}{ OS } \\
\cline { 2 - 4 } & HR & $\mathbf{9 5 \%} \mathbf{C l}$ & P-value \\
\hline Elevated preoperative serum CEA & 2.653 & $0.430-16.379$ & 0.294 \\
Suboptimal debulking & 3.371 & $0.457-24.860$ & 0.233 \\
Advanced stage & 15.925 & $2.745-92.404$ & $\mathbf{0 . 0 0 2}$ \\
\hline
\end{tabular}

Notes: Bold figure indicates statistically significant, $P<0.05$.

Abbreviation: OS, overall survival.

OS

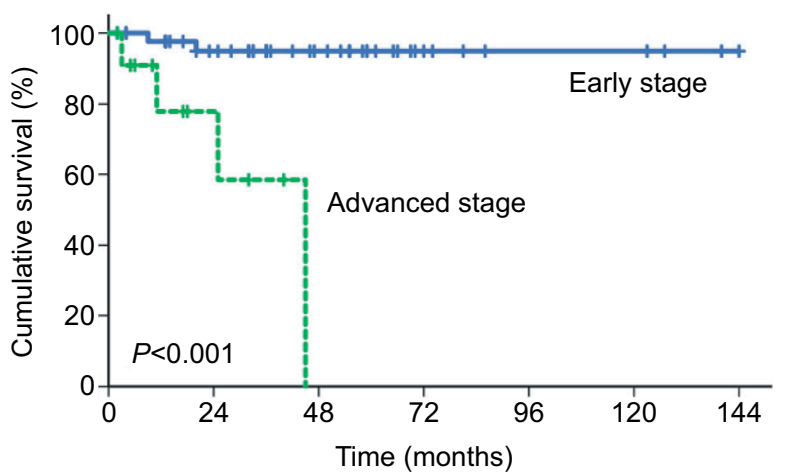

Figure 2 OS curves of patients with primary ovarian mucinous carcinoma according to the FIGO stage (early stage vs advanced stage).

Abbreviations: FIGO, International Federation of Gynecology and Obstetrics; OS, overall survival.

especially for mucin-containing tumors. ${ }^{28}$ In MOC, the reported frequencies of preoperative serum CEA elevation are $41 \%-88 \%{ }^{29,30}$ Our current data suggest that only $17.5 \%$ of patients present with elevated preoperative serum CEA, which is much lower than that reported in previous studies. This phenomenon could be attributed to the inherent selection bias of retrospective studies. Primary MOC patients were excluded from our present study if preoperative measurement of CEA was not available. In fact, the proportion of advanced-stage patients in the current cohort was relatively lower than that in the excluded population $(21.1 \%$ vs $27.9 \%$, respectively; $P=0.427)$. As a result, the frequency of preoperative serum CEA elevation could have been underestimated in this study.

Our results indicate that FIGO stage is an independent prognostic factor of OS in patients with primary MOC, which concurs with a previous study. ${ }^{31}$ In our study, the proportion of patients with elevated CEA was significantly correlated with FIGO stage (early stage vs advanced stage: $8.9 \%$ vs $50.0 \%$, respectively; $P=0.001$ ). On the basis of this, the impact of CEA on survival may be attributed to the correlation between CEA elevation and advanced stage, although elevated preoperative serum $\operatorname{CEA}(P=0.173)$ has no significant effect on OS in the multivariate analysis (Table 5).

Suboptimal cytoreductive surgery is associated with poor survival in patients with EOC. ${ }^{32}$ Our data showed that patients with suboptimal debulking had a worse OS than patients with optimal debulking $(P<0.001)$. In addition, suboptimal debulking was significantly correlated with CEA elevation $(P=0.015)$. This may be explained by the finding that CEA elevation is strongly correlated with advanced-stage MOC cases, in which implantation metastases are more common and thus surgical debulking is more challenging.

Some studies report that elevated serum CEA correlates well with lymph node metastasis of gastric cancer, ${ }^{14}$ breast cancer, ${ }^{33}$ and squamous cell carcinoma of the uterine cervix. ${ }^{34}$ In the present study, lymph node metastasis was found in $4.2 \%(2 / 48)$ of patients. These two patients both had elevated preoperative serum CEA values. One patient recurred with an even higher serum CEA than the preoperative level (48.6 $\mathrm{ng} / \mathrm{mL}$ vs $24.0 \mathrm{ng} / \mathrm{mL}$ ). The other patient had persistently elevated serum CEA and CA125 values, and was lost to follow-up after completing first-line chemotherapy. However, it is difficult to further investigate the association between CEA elevation and lymph node metastasis owing to the limited sample size of the current study.

There is no clear evidence that adjuvant chemotherapy is beneficial in early-stage MOC. ${ }^{35}$ Compared with the serous subtype of EOC, advanced-stage MOC is associated with a poorer response rate to platinum-based chemotherapy and worse survival. ${ }^{36}$ Since the phenotypic appearance and behavior of MOC exhibit some similarities to those of gastrointestinal cancers, gastrointestinal regimens are among the postoperative options for patients with MOC. ${ }^{16,22}$ In our cohort, three patients received gastrointestinal regimens, such as fluorouracil, oxaliplatin, capecitabine, and gemcitabine, combined with platinum regimens as first-line chemotherapy.

\section{Limitations}

There are some limitations of our study that need to be considered when interpreting our data. First, patients were excluded if preoperative CEA values were not available. This may have caused selection bias. In addition, we included only 57 patients with primary MOC due to its rarity. In the future, prospective multi-institute studies will be beneficial to minimize the effect of potential confounding factors.

\section{Conclusion}

Elevation of preoperative serum CEA was strongly correlated with advanced stage in patients with primary MOC and most likely indicated a poor prognosis. Further investigation of 
the intrinsic relationship between CEA and primary MOC is now required.

\section{Acknowledgments}

The authors wish to thank their colleagues in the Department of Pathology for reviewing the histopathological results and their colleagues in the Department of Medical Records for their assistance in retrieving medical charts.

\section{Author contributions}

WL, DC, and KS carried out conception and design of the study. WL contributed to data acquisition. WL and DC performed data analysis and interpretation. WL was involved in manuscript drafting. DC and $\mathrm{KS}$ helped in revising and editing the manuscript. All authors contributed to data analysis, drafting and revising the article, gave final approval of the version to be published, and agree to be accountable for all aspects of the work.

\section{Disclosure}

The authors report no conflicts of interest in this work.

\section{References}

1. Jang JYA, Yanaihara N, Pujade-Lauraine E, et al. Update on rare epithelial ovarian cancers: based on the Rare Ovarian Tumors Young Investigator Conference. J Gynecol Oncol. 2017;28(4):e54.

2. Mueller JJ, Lajer H, Mosgaard BJ, et al. International study of primary mucinous ovarian carcinomas managed at tertiary medical centers. Int J Gynecol Cancer. 2018;28(5):915-924.

3. Hess V, A'Hern R, Nasiri N, et al. Mucinous epithelial ovarian cancer: a separate entity requiring specific treatment. J Clin Oncol. 2004;22(6):1040-1044.

4. Schiavone MB, Herzog TJ, Lewin SN, et al. Natural history and outcome of mucinous carcinoma of the ovary. Am J Obstet Gynecol. 2011;205(5):480.e1-480.e8.

5. Simons M, Ezendam N, Bulten J, Nagtegaal I, Massuger L. Survival of patients with mucinous ovarian carcinoma and ovarian metastases. Int J Gynecol Cancer. 2015;25(7):1208-1215.

6. Ledermann JA, Raja FA, Fotopoulou C, et al. Newly diagnosed and relapsed epithelial ovarian carcinoma: ESMO Clinical Practice Guidelines for diagnosis, treatment and follow-up. Ann Oncol.2013;24 Suppl 6:vi24-vi32.

7. Steffensen KD, Waldstrøm M, Brandslund I, Jakobsen A. Prognostic impact of prechemotherapy serum levels of HER2, CA125, and HE4 in ovarian cancer patients. Int J Gynecol Cancer. 2011;21(6):1040-1047.

8. Tang A, Kondalsamy-Chennakesavan S, Ngan H, et al. Prognostic value of elevated preoperative serum CA125 in ovarian tumors of low malignant potential: a multinational collaborative study (ANZGOG0801). Gynecol Oncol. 2012;126(1):36-40.

9. Bast RC, Urban N, Shridhar V, et al. Early detection of ovarian cancer: promise and reality. Cancer Treat Res. 2002;107:61-97.

10. Harrison ML, Jameson C, Gore ME. Mucinous ovarian cancer. Int $J$ Gynecol Cancer. 2008;18(2):209-214.

11. Duffy MJ, Lamerz R, Haglund C, et al. Tumor markers in colorectal cancer, gastric cancer and gastrointestinal stromal cancers: European group on tumor markers 2014 guidelines update. Int J Cancer. 2014;134(11): 2513-2522.
12. Park BW, Oh JW, Kim JH, et al. Preoperative CA 15-3 and CEA serum levels as predictor for breast cancer outcomes. Ann Oncol. 2008;19(4): 675-681.

13. Okada M, Nishio W, Sakamoto T, et al. Prognostic significance of perioperative serum carcinoembryonic antigen in non-small cell lung cancer: analysis of 1,000 consecutive resections for clinical stage I disease. Ann Thorac Surg. 2004;78(1):216-221.

14. Kochi M, Fujii M, Kanamori N, et al. Evaluation of serum CEA and CA19-9 levels as prognostic factors in patients with gastric cancer. Gastric Cancer. 2000;3(4):177-186.

15. Kurman RJ, Carcangju ML, Herrington CS, Young RH. WHO Classification of Tumours of Female Reproductive Organs. 4th ed. Lyon: IARC; 2014

16. Ledermann JA, Luvero D, Shafer A, et al. Gynecologic Cancer InterGroup (GCIG) consensus review for mucinous ovarian carcinoma. Int J Gynecol Cancer. 2014;24(9 Suppl 3):S14-S19.

17. Vang R, Gown AM, Wu LS, et al. Immunohistochemical expression of $\mathrm{CDX} 2$ in primary ovarian mucinous tumors and metastatic mucinous carcinomas involving the ovary: comparison with CK20 and correlation with coordinate expression of CK7. Mod Pathol. 2006;19(11):1421-1428.

18. Prat J. FIGO Committee on Gynecologic Oncology. Staging classification for cancer of the ovary, fallopian tube, and peritoneum. Int $J$ Gynaecol Obstet. 2014;124(1):1-5.

19. Morgan RJ, Copeland L, Gershenson D, et al. NCCN Ovarian Cancer Practice Guidelines. The National Comprehensive Cancer Network. Oncology. 1996;10(11 Suppl):293-310.

20. Morgan RJ, Alvarez RD, Armstrong DK, et al. Ovarian cancer, version 3.2012. J Natl Compr Canc Netw. 2012;10(11):1339-1349.

21. Morgan RJ, Alvarez RD, Armstrong DK, et al. Ovarian cancer, version 2.2013. J Natl Compr Canc Netw. 2013;11(10):1199-1209.

22. Morgan RJ, Armstrong DK, Alvarez RD, et al. Ovarian Cancer, Version 1.2016, NCCN Clinical Practice Guidelines in Oncology. J Natl Compr Canc Netw. 2016;14(9):1134-1163.

23. Shimada M, Kigawa J, Ohishi Y, et al. Clinicopathological characteristics of mucinous adenocarcinoma of the ovary. Gynecol Oncol. 2009;113(3): 331-334.

24. Seidman JD, Kurman RJ, Ronnett BM. Primary and metastatic mucinous adenocarcinomas in the ovaries: incidence in routine practice with a new approach to improve intraoperative diagnosis. Am J Surg Pathol. 2003;27(7):985-993.

25. Khunamornpong S, Suprasert P, Pojchamarnwiputh S, Na Chiangmai W, Settakorn J, Siriaunkgul S. Primary and metastatic mucinous adenocarcinomas of the ovary: Evaluation of the diagnostic approach using tumor size and laterality. Gynecol Oncol. 2006;101(1): 152-157.

26. Yemelyanova AV, Vang R, Judson K, Wu LS, Ronnett BM. Distinction of primary and metastatic mucinous tumors involving the ovary: analysis of size and laterality data by primary site with reevaluation of an algorithm for tumor classification. Am J Surg Pathol. 2008;32(1): $128-138$.

27. Gold P, Freedman SO. Specific carcinoembryonic antigens of the human digestive system. $J$ Exp Med. 1965;122(3):467-481.

28. Tsai CC, Lin H, Huang EY, et al. The role of the preoperative serum carcinoembryonic antigen level in early-stage adenocarcinoma of the uterine cervix. Gynecol Oncol. 2004;94(2):363-367.

29. Khoo SK, Mackay EV. Carcinoembryonic antigen (CEA) in ovarian cancer: factors influencing its incidence and changes which occur in response to cytotoxic drugs. Br J Obstet Gynaecol. 1976;83(10): $753-759$.

30. Tholander B, Taube A, Lindgren A, Sjöberg O, Stendahl U, Tamsen L. Pretreatment serum levels of CA-125, carcinoembryonic antigen, tissue polypeptide antigen, and placental alkaline phosphatase in patients with ovarian carcinoma: influence of histological type, grade of differentiation, and clinical stage of disease. Gynecol Oncol. 1990;39(1):26-33. 
31. Massad LS, Gao F, Hagemann I, Powell M. Clinical Outcomes among Women with Mucinous Adenocarcinoma of the Ovary. Gynecol Obstet Invest. 2016;81(5):411-415.

32. Nick AM, Coleman RL, Ramirez PT, Sood AK. A framework for a personalized surgical approach to ovarian cancer. Nat Rev Clin Oncol. 2015;12(4):239-245.

33. Wu SG, He ZY, Ren HY, et al. Use of CEA and CA15-3 to predict axillary lymph node metastasis in patients with breast cancer. J Cancer. 2016;7(1):37-41.

34. Huang EY, Huang YJ, Chanchien CC, et al. Pretreatment carcinoembryonic antigen level is a risk factor for para-aortic lymph node recurrence in addition to squamous cell carcinoma antigen following definitive concurrent chemoradiotherapy for squamous cell carcinoma of the uterine cervix. Radiat Oncol. 2012;7:13.

35. Trimbos JB, Parmar M, Vergote I, et al. International Collaborative Ovarian Neoplasm trial 1 and Adjuvant ChemoTherapy In Ovarian Neoplasm trial: two parallel randomized phase III trials of adjuvant chemotherapy in patients with early-stage ovarian carcinoma. $J$ Natl Cancer Inst. 2003;95(2):105-112.

36. Alexandre J, Ray-Coquard I, Selle F, et al. Mucinous advanced epithelial ovarian carcinoma: clinical presentation and sensitivity to platinumpaclitaxel-based chemotherapy, the GINECO experience. Ann Oncol. 2010;21(12):2377-2381.

\section{Publish your work in this journal}

Cancer Management and Research is an international, peer-reviewed open access journal focusing on cancer research and the optimal use of preventative and integrated treatment interventions to achieve improved outcomes, enhanced survival and quality of life for the cancer patient. The manuscript management system is completely online and includes a very quick and fair peer-review system, which is all easy to use. Visit http://www.dovepress.com/testimonials.php to read real quotes from published authors. 